\title{
Análise Discriminante das Relações entre Fatores Estratégicos, Indústria e Desempenho em Organizações Brasileiras Atuantes na Indústria Manufatureira
}

\section{A Discriminant Analysis of the Relationship between Strategic Factors, Industry and Performance in Brazilian Manufacturing Industry Companies}

Carlos Alberto Gonçalves*

Doutor em Administração pela USP. Professor pesquisador do CEPEAD/UFMG, Belo Horizonte/MG, Brasil.

Alexandre Teixeira Dias Doutorando em Administração pelo CEPEAD/UFMG, Belo Horizonte/MG, Brasil.

Reynaldo Maia Muniz Doutor pela Universidad Complutense de Madrid, Espanha. Professor Adjunto do CEPEAD/UFMG, Belo Horizonte/MG, Brasil.

*Endereço: Faculdade de Ciências Econômicas (FACE/UFMG), Av. Antônio Carlos, 6627, Pampulha, Belo Horizonte/MG, 31270-901. E-mail: carlos@ face.ufmg.br 


\title{
RESUMO
}

Com o objetivo de identificar quais os fatores discriminantes do desempenho positivo ou negativo das empresas atuantes em ambiente turbulento, foram estudadas empresas brasileiras inseridas no setor de indústria manufatureira, ativas no período entre 1996 e 2001, caracterizado por intensas alterações nos cenários macroeconômicos nacional e mundial, e coletados dados financeiros e macroeconômicos de fonte secundária. Visando ao entendimento das relações do desempenho das empresas em foco com as diversas variáveis macro e microeconômicas determinantes do ambiente competitivo, da orientação estratégica e do contexto macroeconômico, é proposto um modelo discriminante. Como metodologia de mensuração e análise das relações entre as variáveis dependente e independentes, foi adotado o método estatístico multivariado de análise discriminante. Como resultado foram identificadas as posturas estratégicas de exposição ao risco; o endividamento de longo prazo; e a participação de mercado como discriminantes do resultado das organizações estudadas, considerado em termos de lucro ou prejuízo.

Palavras-chave: orientação estratégica; ambiente; desempenho; competição; análise discriminante.

\begin{abstract}
This paper approaches the question: what are the factors that discriminate between positive and negative companies' performance, when in turbulent environment? Aimed in achieving this goal a study was conducted involving Brazilian companies in manufacturing industry that were active in the period comprised between 1996 to 2001, characterized by intense turbulence in national and worldwide macroeconomic scenes. A discriminant function was proposed to measure and analyze the relationship between the dependent variable and the independent variables, comprised by information about competitive environment, strategic orientation and economic context. As results variables risk exposition, long term debt and market share were identified as the factors that discriminate between positive and negative financial performance.
\end{abstract}

Key words: strategic orientation; environment; performance; competition; discriminant analysis. 


\section{INTRODUÇÃo E OBjetIVOS}

As estratégias organizacionais e seus condicionantes têm sido objeto de estudos desenvolvidos na busca por ampliar o conhecimento acerca dos fatores determinantes do desempenho organizacional, usualmente mensurado em termos financeiros e de participação de mercado. $\mathrm{O}$ aprofundamento das abordagens metodológicas, unido à expansão da base teórica, tem permitido aos pesquisadores analisar o efeito conjunto do ambiente competitivo e da orientação estratégica nos resultados organizacionais, estabelecendo uma estrutura para o entendimento de suas relações. Como exemplo de trabalhos realizados que abordam temas relacionados aos tratados nesta pesquisa, aplicados ao cenário competitivo brasileiro, temos: Brito e Vasconcelos (2003a, 2003b, 2004), Bandeira-de-Mello e Marcon (2004a, 2004b), Dias (2004), Dias, Gonçalves e Coleta (2004) e Dias e Gonçalves (2005).

Duas linhas básicas de referência para o desenvolvimento de pesquisas em estratégia, principalmente com foco na identificação dos fatores que influenciam o desempenho das organizações, são: (a) a abordagem da Organização Industrial, que considera uma maior influência dos fatores externos à organização; e (b) a abordagem da Visão Baseada em Recursos, que coloca maior ênfase nos aspectos internos da organização, tais como a capacidade de maximização da utilização dos recursos e o desenvolvimento de competências essenciais. Neste trabalho não se parte de considerações a priori acerca dessas relações, justificando a proposição de um modelo de pesquisa que englobe aspectos de ambas as abordagens, além de conferir-lhe um caráter exploratório.

Adotando como referência o modelo proposto e desenvolvido por Dias (2004), busca-se neste artigo o entendimento e a identificação dos determinantes estratégicos e ambientais do resultado das organizações, analisados em termos de lucro ou prejuízo, levando-se em consideração sua atuação em ambiente caracterizado por turbulências no cenário macroeconômico, gerador de incertezas quanto à efetividade das ações estratégicas implementadas nas organizações. Para alcançar tal objetivo, foram consideradas para análise empresas brasileiras em atividade no período entre 1996 e 2001, classificadas no setor Indústria Manufatureira.

Como metodologia de análise multivariada das relações entre as variáveis preditoras e a variável foco, foi adotada a análise discriminante, em razão de permitir a identificação dos fatores que apresentam maior capacidade de predição 
em relação ao fenômeno em estudo. Devido à natureza exploratória da pesquisa, não são propostas hipóteses a serem verificadas.

Inicialmente, é apresentado o quadro teórico de referência para o desenvolvimento e análise da pesquisa para ser explicitado, a seguir, o processo metodológico e a análise e interpretação dos dados. Por último são tecidas as considerações finais.

\section{Ponderações dos Quadros Teóricos}

Segundo Ansoff (1975), o gestor deve estar atento aos menores sinais de mudanças no ambiente competitivo, agindo de forma a projetar cenários e estabelecer um plano de ação que permita ajustar os rumos da organização, para que esta não seja pega de surpresa, seja por ameaças, que podem custar sua continuidade, seja por oportunidades, que podem valer uma posição de mercado favorável. Dessa forma, é relevante para o gestor estar atento ao contexto competitivo em que sua organização se insere, tema tratado adiante.

\section{Teoria da Competição e Estratégia}

Tendo em vista o constante embate entre as organizações em busca de um desempenho superior, tanto em termos financeiros, quanto em termos de participação de mercado, diversos autores abordaram as variadas formas de configuração da competição, basicamente com referência a aspectos microeconômicos. Dentre esses, Barney (1986) buscou estabelecer uma referência que integre estas diversas abordagens, relacionando as três principais correntes microeconômicas no campo da competição: (a) Organização Industrial - Industrial Organization Economics - considera que os retornos das empresas são determinados pela estrutura do setor em que atuam e que as características de tal setor que mais influenciam tais retornos são a existência e a intensidade de barreiras à entrada, a quantidade e o tamanho relativo das empresas, o nível de diferenciação de produtos ofertados e a elasticidade da demanda do setor (Porter, 1980, 1981). Um dos aspectos marcantes dessa abordagem é o paradigma SCP (Structure, Conduct, Performance) que relaciona estrutura setorial (structure), estratégia (conduct) e desempenho (performance), sendo que os dois últimos são determinados ou delineados pela primeira (Barney, 1986; Hunt, 2000). (b) Abordagem Chamberliniana, Chamberlinian economics, busca entender e explicar as estratégias organizacionais; mas, diferentemente da primeira, com foco nas características individuais únicas e nas capacidades das empresas, 
identificando o impacto dessas individualidades nas estratégias formuladas e nos resultados delas decorrentes. Para Chamberlin, citado por Barney (1986), a competição intra-setor sempre ocorre entre empresas com diferentes recursos e características, sendo tratada a questão dos aspectos idiossincrásicos da organização, os quais permitem a alteração da estrutura setorial por meio da implementação da estratégia empresarial, beneficiando somente a própria empresa. Assim, dentre outros aspectos considerados por essa abordagem, a heterogeneidade das empresas atuantes no setor constitui uma fonte de vantagens competitivas. De acordo com a abordagem Chamberliniana, com ênfase na questão da competição monopolística, as empresas devem esforçar-se por escolher as estratégias que maximizem os resultados da exploração de suas capacidades individuais e de seus diferenciais, habilitando-se a obter retornos econômicos relativamente elevados e a manter sua capacidade competitiva e (c) Abordagem Schumpeteriana, Schumpeterian economics, de acordo com Barney (1986), considera os aspectos relacionados à incerteza competitiva, servindo de referência para pesquisas que se propõem compreender as reações das organizações em face das configurações de mercado, que irão requerer o desenvolvimento de novas capacidades, estabelecendo os padrões tecnológicos e mercadológicos de competição, quais os recursos e capacidades que são estratégicos para o sucesso das empresas e quais não apresentam peso significativo para os resultados organizacionais. Ainda conforme o autor, reações tardias, assim como antecipações equivocadas, podem minar a capacidade competitiva das empresas, além de criar um cenário difícil de ser revertido.

Em sua proposta de integração das abordagens da competição, Barney (1986) ressaltou que o setor de atividade é a unidade de análise das três correntes de pensamento e que não há como considerar a questão da competição interfirmas somente com base em uma das três correntes de análise, pois as três se complementam: a abordagem Schumpeteriana trata da incerteza do mercado; a abordagem da Organização Industrial tem como foco a estrutura e os relacionamentos do mercado; a abordagem Chamberliniana se baseia nas características únicas e nos recursos diferenciados para identificar os aspectos que determinam as estratégias competitivas das empresas.

\section{Estratégia e Ambiente Competitivo}

Child (1974, 1975) definiu o ambiente em que uma organização está inserta conforme sua variabilidade e sua complexidade. Por variabilidade entende-se a ocorrência de mudanças que são difíceis de prever, envolvendo diferenças significativas em relação às condições ambientais anteriormente identificadas, gerando, conseqüentemente, incerteza quanto ao futuro. Associa complexidade à amplitude e à heterogeneidade das atividades externas à organização, as quais 
são relevantes para o planejamento e para as operações, constituindo fonte de informações que devem ser monitoradas pela organização. Tal abordagem do ambiente competitivo está em alinhamento com os achados de Duncan (1972), que afirmou que o nível de incerteza a ser gerenciado no processo de tomada de decisões apresenta maior relação com o grau de variabilidade no ambiente do que com o seu nível de complexidade.

Chrisman, Hofer e Boulton (1988) afirmaram que a estratégia competitiva das organizações pode ser descrita com base em três fatores: a) escopo configuração das interações entre a organização e seu ambiente, descrevendo seu domínio de atuação; b) armas competitivas - as principais formas pelas quais a organização aplica suas capacitações e seus recursos para atender às exigências do ambiente e criar vantagens competitivas duradouras; e c) diferenciação de segmento - utilização de tipos diferentes de armas competitivas em diferentes segmentos de mercado.

Hatten e Schendel ${ }^{(1)}$, citados por Kim e Lim (1988), concluíram que empresas atuantes em um mesmo setor adotam posturas competitivas diferenciadas, em virtude de elaborarem abordagens ambientais em uma base individual, ou única, que também serve de referência para as decisões acerca da alocação de recursos.

\section{Competição Baseada em Recursos}

Hunt e Morgan (1995, 1996, 1997) e Hunt $(1997,2000)$, ao abordarem a questão da competição, propuseram a Teoria da Vantagem em Recursos - R-A Theory, que tem por premissas: (a) a inovação e a aprendizagem organizacionais são endógenas à competição; (b) o acesso à informação é imperfeito; diferentes empresas têm acesso às informações a custos diferentes e em momentos diversos; e (c) as políticas e as instituições públicas afetam diretamente o desempenho econômico das organizações. Outro aspecto fundamental da teoria da vantagem em recursos é considerar que o processo de seleção das organizações, pelo mercado em que atuam, baseia-se na competição pelos recursos que garantam o estabelecimento de uma vantagem perante seus concorrentes, evidenciando a fundamentação da teoria na visão baseada em recursos. Tal fundamentação, somada à heterogeneidade da demanda e ao acesso imperfeito à informação, leva à diversificação do tamanho das empresas, do escopo de suas atividades e dos níveis de lucratividade, não somente entre setores diferenciados, mas também internamente aos diversos setores de atividade.

Conforme pode ser observado, há ampla diversidade de perspectivas acerca dos fatores que influenciam o desempenho das organizações, com predominância de trabalhos que tratam dos aspectos externos à organização, principalmente no 
tocante à dinâmica do seu mercado de atuação, ou dos aspectos internos, com especial atenção voltada para os trabalhos referenciados na abordagem da Visão Baseada em Recursos. Outros trabalhos, tais como os de Roquebert, Phillips e Westfall (1996), baseado nas pesquisas de Schmalensee (1985) e de Rumelt (1991), de Mauri e Michaels (1998) e de Hawawini, Subramanian e Verdin (2003), buscam desvendar as relações entre fatores oriundos da indústria e pontos específicos das empresas, principalmente no tocante à alocação de recursos e escolhas estratégicas, e desses conjuntamente com o desempenho das organizações.

Apresentadas as bases teóricas nas quais se referencia a abordagem da questão de pesquisa proposta, é apresentado, na próxima seção, o modelo base de pesquisa, cujas dimensões, categorias e expressões foram construídas levando-se em consideração a busca pela expressão dos aspectos teóricos tratados, norteadores do trabalho.

\section{Processo Metodológico}

Com o objetivo de estudar os aspectos determinantes do desempenho das organizações, quando atuantes em um ambiente turbulento, Dias (2004) propôs o modelo base deste estudo, composto pelas dimensões, respectivas categorias e expressões, abordadas a seguir, sendo considerados os aspectos relacionados ao desempenho da organização e seu relacionamento com o ambiente competitivo e a orientação estratégica da empresa, os quais são influenciados pelo contexto macroeconômico. O construto desempenho é definido com base nas categorias participação de mercado (MS) e rentabilidade (ROA). Tomando por base os objetivos propostos nesta pesquisa e destacando que não se trata da replicação do trabalho desenvolvido por Dias (2004), a variável participação de mercado (MS) passou a ser considerada como preditora do desempenho positivo ou negativo (lucro ou prejuízo) das empresas analisadas. Já o contexto é mensurado em função dos condicionantes macroeconômicos expressos pelo setor (SETOR), relativo ao desempenho anual do setor de atividade como um todo, do subsetor (SSETOR), referente ao desempenho anual do subsetor e do desempenho anual da própria empresa (FIRMA). O ambiente competitivo é abordado por meio das suas características de dinamismo (DINAM) - variação do PIB do subsetor, comparada à variação do PIB do país e rivalidade (RIVAL) - relação entre o número de concorrentes no subsetor e o total de empresas atuantes no setor pesquisado, incluindo, assim, os concorrentes potenciais. 
A orientação estratégica é identificada pelo relacionamento entre as variáveis: postura defensiva ou prospectiva (POSTURA) - reflete o comportamento dos gestores segundo a tipologia de Miles, Snow, Meyer e Coleman (1978, p. 550), mensurado em relação à ênfase dada à redução de custos; foco no longo prazo (FOCO), expressa considerações de alocação de recursos em termos de tempo; efetividade (longo prazo) em comparação com eficiência (curto prazo); e exposição ao risco (EXPRISCO1 e EXPRISCO2) - risco incorrido pela empresa refletido nas decisões acerca da alocação de recursos, em detrimento de retornos financeiros. A operacionalização das variáveis é representada na Tabela 1.

\section{Tabela 1: Variáveis do Modelo}

\begin{tabular}{|c|c|c|}
\hline CATEGORIA & VARIÁVEL & FORMA DE CÁLCULO \\
\hline \multicolumn{3}{|c|}{ VARIÁVEL DEPENDENTE } \\
\hline Rentabilidade & Retorno sobre o ativo (ROA) & Lucro líquido/Ativo total \\
\hline \multicolumn{3}{|c|}{ VARIÁVEIS INDEPENDENTES } \\
\hline Participação de mercado & Participação de mercado (MS) & Faturamento bruto no período/ PIB do setor \\
\hline Setor & Crescimento do setor (SETOR) & Variação anual do PIB setorial \\
\hline Subsetor & $\begin{array}{l}\text { Crescimento do subsetor } \\
\text { (SSETOR) }\end{array}$ & Variação anual do PIB do subsetor \\
\hline Firma & Resultado da empresa (FIRMA) & Variação anual da receita bruta \\
\hline $\begin{array}{l}\text { Postura defensiva ou } \\
\text { prospectiva }\end{array}$ & Gestão de custos (POSTURA) & $\begin{array}{l}\text { Variação anual da relação entre Custo do Produto } \\
\text { Vendido e Faturamento Bruto }\end{array}$ \\
\hline Endividamento de longo prazo & $\begin{array}{l}\text { Perfil do endividamento de longo } \\
\text { prazo (ENDIVLP) }\end{array}$ & $\begin{array}{l}\text { Relação entre financiamentos de longo prazo e } \\
\text { total de financiamentos em base anual }\end{array}$ \\
\hline Exposição ao risco & $\begin{array}{l}\text { Alocação de recursos na atividade } \\
\text { produtiva em detrimento de } \\
\text { retornos financeiros (EXPRISCO1, } \\
\text { EXPRISCO2) }\end{array}$ & $\begin{array}{l}\text { Relação entre recursos investidos na compra de } \\
\text { ativos fixos e lucro líquido no período em base } \\
\text { anual (ExpRisco1) } \\
\text { Aumento do investimento no permanente em } \\
\text { base anual (ExpRisco2) }\end{array}$ \\
\hline Dinamismo & $\begin{array}{l}\text { Crescimento do subsetor em } \\
\text { relação ao crescimento da } \\
\text { economia nacional (DINAM) }\end{array}$ & $\begin{array}{l}\text { Variação anual do PIB do subsetor, comparada à } \\
\text { variação anual do PIB nacional }\end{array}$ \\
\hline Rivalidade & $\begin{array}{l}\text { Intensidade da concorrência } \\
\text { (RIVAL) }\end{array}$ & $\begin{array}{l}\text { Relação entre o número de concorrentes no } \\
\text { subsetor e o total de empresas atuantes no setor } \\
\text { pesquisado em base anual }\end{array}$ \\
\hline
\end{tabular}

Fonte: adaptado de Dias (2004).

Considerando o objetivo geral estabelecido para a pesquisa, qual seja, contribuir para o entendimento e a identificação dos determinantes estratégicos e ambientais do resultado das organizações, analisados em termos de lucro ou prejuízo, e levando-se em consideração sua atuação em um ambiente turbulento, para 
consecução dos trabalhos foram utilizados dados secundários de várias fontes, tais como Economática ${ }^{\circledR}$, IBGE e IPEA, relativos ao desempenho econômicofinanceiro e alocação de recursos por parte das empresas, dados setoriais e informações macroeconômicas, que foram trabalhados de forma a permitir a operacionalização dos indicadores e a mensuração das suas relações.

\section{Método}

Para atingir o objetivo de pesquisa foi utilizada a técnica estatística de análise discriminante, por meio do software estatístico SPSS ${ }^{\circledR}$, a qual consiste em análise de um modelo em que a variável dependente é categórica, que em muitos casos consiste em dois ou mais grupos de classificação, e as variáveis independentes são métricas ou de natureza intervalar. A técnica aplicada na pesquisa foi a análise discriminante de dois grupos, pois a variável dependente, envolvida na análise (ROA), tem duas categorias: lucro e prejuízo. Quando duas classificações são envolvidas, a técnica é referida aos dois grupos de análise discriminante, sendo apropriada para testar as hipóteses de que as médias dos grupos das variáveis independentes localizadas para dois ou mais grupos são iguais. Estas médias dos valores dos escores discriminantes dos grupos se referem aos centróides deles, existindo tantos centróides quantos são os grupos; uma comparação entre esses mostra quanto estão separados os grupos durante o teste da função discriminante.

A técnica tem como objetivos:

. Estabelecer funções discriminantes que melhor discriminem as categorias de variável dependente (ROA: lucro e prejuízo).

- Analisar se existem diferenças significativas entre os grupos, em relação às variáveis independentes.

. Identificar as variáveis independentes que mais contribuem para as diferenças entre os grupos.

- Classificar os casos em um dos grupos de acordo com os valores das variáveis independentes.

Analisar com precisão a classificação.

A equação geral do modelo discriminante é:

$\mathrm{D}=\mathrm{b}_{0}+\mathrm{b}_{1} \mathrm{X}_{1}+\mathrm{b}_{2} \mathrm{X}_{2}+\mathrm{b}_{3} \mathrm{X}_{3}+\mathrm{b}_{4} \mathrm{X}_{4}+\ldots+\mathrm{b}_{\mathrm{k}} \mathrm{X}_{\mathrm{k}}$ Equação (1) 
Onde D é o escore discriminante; $\mathrm{b}$ é o coeficiente ou o peso discriminante; $\mathrm{X}$ representa a variável independente, $\mathrm{D}$ é uma variável categórica e $\mathrm{X}_{1}, \mathrm{X}_{2}, \mathrm{X}_{3}$, $\mathrm{X}_{4}, \ldots, \mathrm{X}_{\mathrm{k}}$ são variáveis intervalares e / ou razão.

A discriminação é realizada por meio da estimação dos pesos $\left(b_{k}\right)$ de cada variável, com o objetivo de maximizar a variância intra e entre grupos, de modo que os grupos se distingam o máximo possível dos valores da função discriminante. $\mathrm{Na}$ análise, multiplica-se cada variável independente por seu peso correspondente e somam-se esses produtos; o resultado é um escore discriminante composto para cada indivíduo considerado na análise. Apurando-se a média desses, obtêmse os centróides (Aaker, Kumar, \& Day, 2001; Hair, Anderson, Tatham, \& Black, 1998; Malhotra, 2001).

\section{Comentários de Pré-Condições}

Segundo Mingoti (2003), o princípio da máxima verossimilhança pode ser aplicado mesmo em situações em que a distribuição amostral envolvida não é normal, apesar dos softwares estatísticos usuais construírem uma função discriminante apenas para dados com distribuição normal. Devido a esse aspecto, deve-se tomar cuidado, ao fazer a análise discriminante computacionalmente, pois os resultados obtidos com a análise terão validade se as variáveis originais forem normais ou se permitirem uma aproximação para a distribuição normal. Apesar dessa pré-condição de normalidade dos dados, conscientemente foi feita a análise discriminante com os dados originais não normalizados.

\section{Estratégia de Análise}

Para realização do processamento dos dados e construção da análise dos resultados, foram adotados os seguintes procedimentos.

. Análise Discriminante com os dados originais, análise com os dados não normalizados.

- Análise Discriminante com os dados transformados, desenvolvimento do modelo discriminante, normalizando os dados por meio do Teorema Central do Limite.

- Validação com amostras estratificadas, para convalidar os dois modelos anteriores. 


\section{Análise e Interpretação dos Dados}

\section{Aquisição e Representação dos Dados}

Os dados relativos às organizações atuantes no setor selecionado para estudo foram coletados por meio da identificação, no banco de dados Economática ${ }^{\circledR}$, das empresas que atendem aos critérios a seguir apresentados. Os casos foram identificados e as variáveis selecionadas período a período, visando garantir a qualidade dos dados e a integridade da amostra. Quanto às informações setoriais, os dados relativos ao PIB nacional, ao desempenho do setor, dos subsetores em análise e a quantidade de empresas atuantes no setor analisado, todos em base anual, foram obtidos no Instituto Brasileiro de Geografia e Estatística, IBGE, fazendo-se uso de consulta direta à base de dados disponibilizada pela instituição em seu web site. Quanto aos critérios de seleção das empresas a serem incluídas na base de dados, foram definidas como unidades de análise empresas brasileiras de capital aberto, ativas no período de 1996 a 2001, inseridas na categoria, 31 Indústria Manufatureira, segundo a classificação 3 dígitos do padrão North American Industry Classification System, NAICS, adotado pela base de dados Economática ${ }^{\circledR}$ e que apresentaram receita bruta positiva e maior que $\mathrm{R} \$ 10$ milhões em pelo menos 3 dos anos analisados, resultando num total de 146 empresas.

Assim, foram selecionados 667 casos, considerando o conjunto de informações por empresa e por ano analisado, sendo 46 descartados por caracterizarem casos discrepantes, em razão de se enquadrarem, simultaneamente, nos critérios de identificação de outliers uni e multivariados propostos por Hair et al. (1998). Como outliers univariados foram identificados os casos cujos valores da variável em análise distanciam 3 desvios-padrão da média amostral. Já os outliers multivariados foram identificados por meio da análise da Distância de Mahalanobis - D2, em nível de significância de 0,001, considerado muito conservador por Hair et al. (1998). Tais casos foram analisados individualmente, considerando-se a natureza da variável, a sua forma de mensuração e os objetivos da pesquisa, sendo excluídos da amostra aqueles que se caracterizaram como possível origem de prejuízos para o resultado da pesquisa, chegando-se a uma amostra de 621 casos.

No processo de análise foi utilizada a variável ROA (Retorno sobre o ativo) como dependente, categorizada em dois grupos (lucro e prejuízo), e como independentes as variáveis SSETOR (Crescimento do subsetor), SETOR (Crescimento do setor), DINAM (Dinamismo), RIVAL (Intensidade da concorrência), POSTURA (Gestão de custo), EXPRISCO1 (Exposição ao risco 1), EXPRISCO2 (Exposição ao risco 2), MS (Participação de mercado), FIRMA (Resultado da empresa), ENDIVLP (Endividamento de longo prazo) e ENDIVCP 
(Endividamento de curto prazo); as duas últimas representam informações similares às disponibilizadas pela variável FOCO do modelo de Dias (2004).

Para garantia da qualidade dos resultados apurados, foi realizado o teste de correlação entre as variáveis ENDIVLP e ENDIVCP. A correlação entre as variáveis é negativa (coeficiente de correlação de Pearson $=-0,774$ ) e estatisticamente significante a $1 \%(n=621)$, razão pela qual apenas a variável ENDIVLP foi referenciada na análise.

\section{Análise Discriminante com os Dados Originais}

Por meio do Teste de Igualdade de Médias obteve-se como resultado que apenas as variáveis SETOR, EXPRISCO1, ENDIVLP e MS são estatisticamente significativas $(\mathrm{p}<0,05)$ na diferenciação dos dois grupos: 0 (prejuízo) e 1 (lucro).

De acordo com a Tabela 2, os coeficientes padronizados indicam que a variável EXPRISCO1 é o prognosticador mais importante na discriminação entre os grupos, seguido pelas variáveis ENDIVLP, MS e SETOR, em relação a seus valores absolutos. Os coeficientes não-padronizados, Tabela 3 , indicam que duas variáveis (EXPRISCO2 e FIRMA) não discriminam a função, ou seja, não influenciam o sinal da variável dependente ROA. Em relação à variável FIRMA, o resultado pode ser explicado em função de que esta mede apenas a quantidade de venda de uma empresa não especificando a quantidade de gastos.

\section{Tabela 2: Coeficientes Padronizados da Função Discriminante}

\begin{tabular}{l|r|r|r|r}
\hline \hline \multirow{2}{*}{ Variáveis } & \multicolumn{5}{|c}{ Dados } \\
\cline { 2 - 5 } & Originais & Transformados & 1a. Amostra de Análise & 2a. Amostra de Análise \\
\hline SSETOR & 0,061 & 0,199 & 0,141 & $-0,009$ \\
SETOR & 0,245 & $* * *-\mathbf{0 , 4 5 3}$ & 0,298 & 0,149 \\
DINAM & $-0,078$ & $* * \mathbf{0 , 4 5 9}$ & $-0,185$ & 0,005 \\
RIVAL & 0,193 & 0,008 & 0,301 & 0,066 \\
POSTURA & 0,084 & 0,358 & 0,168 & $-0,025$ \\
EXPRISCO2 & 0,044 & 0,212 & 0,260 & $-0,015$ \\
EXPRISCO1 & $* \mathbf{0 , 8 9 2}$ & $-0,045$ & $* \mathbf{0 , 8 2 2}$ & $* \mathbf{0 , 9 2 9}$ \\
ENDIVLP & $* * \mathbf{0 , 3 4 4}$ & $* \mathbf{0 , 6 9 6}$ & $* * \mathbf{0 , 3 2 2}$ & $* * \mathbf{0 , 3 7 5}$ \\
MS & $* * * \mathbf{0 , 2 5 6}$ & 0,370 & $* * * \mathbf{0 , 3 0 4}$ & $* * * \mathbf{0 , 1 8 4}$ \\
FIRMA & $-0,157$ & 0,317 & $-0,079$ & $-0,183$ \\
\hline \hline
\end{tabular}

Fonte: Dados da pesquisa.

Legenda:

* - $1^{\text {a }}$. variável com maior coeficiente.

** $-2^{\text {a }}$. variável com maior coeficiente.

$* * *-3^{\mathrm{a}}$. variável com maior coeficiente. 
Tabela 3: Coeficientes Não-padronizados da Função Discriminante

\begin{tabular}{l|r|r|r|r}
\hline \hline \multirow{2}{*}{ Variáveis } & \multicolumn{5}{|c}{ Dados } \\
\cline { 2 - 5 } & Originais & Transformados & 1a. Amostra de Análise & 2a. Amostra de Análise \\
\hline SSETOR & 0,002 & 0,031 & 0,005 & 0,000 \\
SETOR & 0,030 & $-0,208$ & 0,037 & 0,018 \\
DINAM & $-0,024$ & 0,497 & $-0,057$ & 0,002 \\
RIVAL & 4,344 & 0,656 & 6,751 & 1,497 \\
POSTURA & 0,539 & 9,265 & 0,998 & $-0,180$ \\
EXPRISCO & & & & \\
2 & $\mathbf{0 , 0 0 0}$ & $\mathbf{0 , 0 0 0}$ & $\mathbf{0 , 0 0 0}$ & $\mathbf{0 , 0 0 0}$ \\
EXPRISCO & & & & 0,467 \\
1 & 0,463 & $-0,090$ & 1,079 & 1,299 \\
ENDIVLP & 1,171 & $-9,690$ & 0,080 & 0,042 \\
MS & 0,062 & 0,344 & $\mathbf{0 , 0 0 0}$ & $\mathbf{0 , 0 0 0}$ \\
FIRMA & $\mathbf{0 , 0 0 0}$ & $\mathbf{0 , 0 0 1}$ & $-1,686$ & $-1,119$ \\
Constante & $-1,427$ & 4,760 & & \\
\hline \hline
\end{tabular}

Fonte: Dados da pesquisa.

Consultando a Tabela 4, pode-se identificar as correlações combinadas dentro de grupos entre variáveis discriminantes e funções discriminantes canônicas padronizadas (variáveis ordenadas pelo tamanho da correlação dentro da função). Com a matriz estrutural tem-se em ordem de grandeza, a correlação simples entre os preditores e a função discriminante (Malhotra, 2001). A variável EXPRISCO1 possui maior correlação com a função discriminante, seguida pelas variáveis ENDIVLP, MS e SETOR.

\section{Tabela 4: Matriz Estrutural}

\begin{tabular}{l|c}
\hline \hline \multicolumn{1}{c|}{ Variáveis } & Função 1 \\
\hline EXPRISCO1 (Exposição ao Risco 1) & 0,848 \\
ENDIVLP (Endividamento de Longo Prazo) & 0,319 \\
MS (Participação de Mercado) & 0,213 \\
SETOR (Crescimento do Setor) & 0,182 \\
FIRMA (Resultado da Empresa) & $-0,098$ \\
SSETOR (Crescimento do Subsetor) & 0,078 \\
DINAM (Dinamismo) & 0,076 \\
POSTURA (Gestão de Custo) & 0,076 \\
RIVAL (Intensidade da Concorrência) & 0,071 \\
EXPRISCO2 (Exposição ao Risco 2) & 0,008 \\
\hline \hline
\end{tabular}

Fonte: Dados da pesquisa. 
Segundo Malhotra (2001), os centróides dos grupos fornecem o valor da função discriminante calculado com base nas médias dos grupos. Para o grupo 0 (prejuízo) apurou-se valor negativo $(-0,586)$, ao passo que para o grupo 1 (lucro) foi apurado valor positivo $(0,416)$. Os sinais dos coeficientes associados aos prognosticadores indicam o tipo de correlação das variáveis independentes em relação à dependente. Com base nos resultados apurados, o ROA positivo de uma empresa pode resultar de maior exposição ao risco, maior perfil de endividamento de longo prazo, maior participação de mercado e maior crescimento do setor.

De acordo com a Tabela 5, identifica-se que o grupo 0 (prejuízo) apresenta maior porcentagem de classificações corretas (81\%) do que o grupo lucro (67\%).

Tabela 5: Resultados da Classificação ${ }^{a}$

\begin{tabular}{|c|c|c|c|c|c|}
\hline & & \multirow[b]{2}{*}{ ROA } & \multicolumn{2}{|c|}{ Grupos Preditos } & \multirow{2}{*}{ Total } \\
\hline & & & 0,00 & 1,00 & \\
\hline \multirow{3}{*}{ Original } & $\mathrm{N}^{\circ}$ de casos & 0,00 & $\begin{array}{l}209 \\
121\end{array}$ & $\begin{array}{c}49 \\
242\end{array}$ & $\begin{array}{l}258 \\
363\end{array}$ \\
\hline & \multirow{2}{*}{$\%$} & 0,00 & 81 & 19 & 100 \\
\hline & & 1,00 & 33 & 67 & 100 \\
\hline
\end{tabular}

${ }^{a}$ percentagem de casos grupados classificados corretamente: $72,6 \%$

Fonte: Dados da pesquisa.

Devido ao resultado obtido, em que duas variáveis não influenciaram a equação discriminante (EXPRISCO2 e FIRMA), uma nova análise discriminante foi realizada, excluindo-as do modelo, mas não foram obtidas modificações significantes nas porcentagens dos resultados da classificação. A equação do modelo de análise discriminante para os dados analisados é:

$$
\begin{aligned}
& \text { ROA }=-1,427+0,002 * \text { SSETOR }+0,030 * \text { SETOR }-0,024 * \text { DINAM + } \\
& 4,344 * \text { RIVAL }++0,539 * \text { POSTURA + 0,463*EXPRISCO }+1,171 * \text { ENDIVLP } \\
& +0,062 * \text { MS }
\end{aligned}
$$

Com o objetivo de alcançar melhor resultado nas classificações, foi adotado o procedimento de normalização dos dados, sendo que ao se adotar os métodos tradicionais de transformação de dados não foi obtido sucesso. Em razão de tal insucesso, foi aplicado o Teorema Central do Limite com nível de significância de 5\%. Assim, cada variável ficou com 615 casos (para se obter amostras com o mesmo número de casos), sendo estes divididos em 41 amostras compostas de 15 dados. Para cada amostra foi retirada a média, totalizando 41 médias para cada variável. Para a maioria das variáveis obteve-se sucesso na normalização, exceto para EXPRISCO2, MS e FIRMA, ou seja, de 11 variáveis apenas 3 não foram obtidas as normalizações. Com os dados transformados, processou-se a 
análise discriminante novamente, a fim de se verificar a ocorrência de alguma diferença nos resultados de classificação entre a análise dos dados originais e dos dados transformados.

\section{Análise Discriminante com os Dados Transformados}

Para identificar os coeficientes das variáveis estatisticamente significativos ( $\mathrm{p}<0,05)$, foi realizado o Teste de Igualdade de Médias, concluindo que SETOR e ENDIVLP são determinantes na diferenciação dos dois grupos - 0 (prejuízo) e 1 (lucro), assim como na análise dos dados originais.

De acordo com a Tabela 2, os coeficientes padronizados indicam que a variável ENDIVLP é o prognosticador mais importante na discriminação entre os grupos, em valor absoluto, seguido pelas variáveis DINAM, SETOR e MS. Observando os coeficientes não-padronizados, Tabela 3, pode-se afirmar que duas variáveis (EXPRISCO2 e FIRMA) continuam não discriminando a função, ou seja, não influenciam o sinal da variável dependente ROA (prejuízo e lucro).

Analisando pela Tabela 6, verifica-se que ocorrem algumas discordâncias em relação às variáveis de maior correlação com a função discriminante, pois a ordem de grandeza das variáveis não coincide com a encontrada nos coeficientes padronizados.

\section{Tabela 6: Matriz Estrutural}

\begin{tabular}{l|c}
\hline \hline \multicolumn{1}{c|}{ Variáveis } & Função 1 \\
\hline SETOR (Crescimento do Setor) & $-0,549$ \\
ENDIVLP (Endividamento de Longo Prazo) & $-0,541$ \\
SSETOR (Crescimento do Subsetor) & 0,303 \\
DINAM (Dinamismo) & 0,243 \\
POSTURA (Gestão de Custo) & 0,234 \\
FIRMA (Resultado da Empresa) & 0,177 \\
RIVAL (Intensidade da Concorrência) & 0,150 \\
MS (Participação de Mercado) & 0,107 \\
EXPRISCO2 (Exposição ao Risco 2) & 0,107 \\
EXPRISCO1 (Exposição ao Risco 1) & 0,010 \\
\hline \hline
\end{tabular}

Fonte: Dados da pesquisa.

Os centróides dos grupos fornecem o valor da função discriminante calculado por meio das médias dos grupos: o grupo 0 (prejuízo) tem valor positivo $(0,494)$, enquanto o grupo 1 (lucro) apresenta valor negativo $(-0,953)$. 
Na Tabela 7 observa-se que os dados utilizados para a classificação dos grupos 0 e 1 foram considerados $50 \%$ para cada grupo, indicando uma boa porcentagem para a análise.

Tabela 7: Probabilidades Prévias para os Grupos

\begin{tabular}{c|c|c|c}
\hline \hline \multirow{2}{*}{ ROA } & \multirow{2}{*}{ Prévia } & \multicolumn{2}{|c}{ Casos Usados na Análise } \\
\cline { 3 - 4 } & & Não ponderados & Ponderados \\
\hline 0,00 & 0,500 & 27 & 27 \\
1,00 & 0,500 & 14 & 14 \\
Total & 1,000 & 41 & 41 \\
\hline \hline
\end{tabular}

Fonte: Dados da pesquisa.

Analisando a Tabela 8, identifica-se que o grupo 0 (prejuízo) apresenta maior porcentagem de classificações corretas (74\%), comparado ao grupo 1 (lucro) com 71\%. Portanto, pode-se dizer que quando um caso está classificado em prejuízo, significa que a chance de tal classificação estar correta é maior do que se estiver classificado em lucro, devido aos resultados das porcentagens. Em relação à percentagem de casos grupados classificados corretamente, esta é considerada boa.

Tabela 8: Resultados da Classificação ${ }^{\mathrm{a}}$

\begin{tabular}{l|c|c|c|c|c}
\hline \hline \multicolumn{2}{c}{} & & \multicolumn{2}{c|}{ Grupos Preditos } & \multirow{2}{*}{ Total } \\
\cline { 4 - 5 } \multicolumn{2}{c}{} & ROA & 0,00 & 1,00 & \\
\hline \multirow{4}{*}{ Original } & \multirow{3}{*}{$N^{\circ}$ de casos } & 0,00 & 20 & 7 & 27 \\
\cline { 3 - 5 } & & 1,00 & 4 & 10 & 14 \\
\cline { 3 - 5 } & \multirow{2}{*}{$\%$} & 0,00 & 74 & 26 & 100 \\
& & 1,00 & 29 & 71 & 100 \\
\hline \hline
\end{tabular}

a percentagem de casos grupados classificados corretamente: $73,2 \%$

Fonte: Dados da pesquisa.

Comparando os resultados de percentagens obtidos, referentes aos casos originais e aos casos transformados, pode-se dizer que não há diferença significativa entre eles $(72,6 \%$ e $73,2 \%$, respectivamente). Isto indica que, para a amostra, a normalidade dos dados não apresenta influência direta em relação aos resultados da classificação. De acordo com os resultados obtidos, a equação do modelo de análise discriminante para os dados analisados é:

$$
\begin{aligned}
& \text { ROA }=4,760+0,031 * \text { SSETOR }-0,208 * \text { SETOR }+0,497 * \text { DINAM + } \\
& 0,656 * \text { RIVVAL }++9,265 * \text { POSTURA - 0,090*EXPRISCO }-9,690 * \text { ENDIVLP } \\
& +0,344 * \text { MS + 0,001*FIRMA }
\end{aligned}
$$




\section{Validação com Amostras Estratificadas}

$\mathrm{Na}$ busca de maior definição da resposta e de maior percentagem de casos grupados, classificados corretamente, foi realizada nova análise discriminante, dividindo a amostra em metades, de maneira aleatória. Segundo Malhotra (2001), uma das metades da amostra, chamada amostra de estimação ou amostra de análise, é usada para estimar a função discriminante. A outra metade, chamada amostra de validação ou amostra retida (holdout), é reservada para validar a função discriminante. Após a estimação dos parâmetros, invertem-se os papéis das subamostras e repete-se a análise em processo de dupla validação cruzada.

Para dividir a amostra foram selecionados, de maneira aleatória, 310 dos 621 casos, para a primeira amostra, e 311 para a segunda. A seguir são apresentados os resultados relativos à primeira metade.

\section{Análise Discriminante para a Primeira Metade da Amostra}

Com a análise discriminante dos dados da primeira amostra retirada da população, quanto à significância em relação ao Teste de Igualdade de Médias, têm-se as variáveis SETOR, EXPRISCO1, ENDIVLP e MS, resultado semelhante ao obtido por meio da análise dos dados originais (nãonormalizados).

Como pode ser observado na Tabela 2 , os coeficientes padronizados indicam que a variável EXPRISCO1 é o prognosticador de maior importância na discriminação entre os grupos. Esta é seguida, por ordem de grandeza (em módulo), pelas variáveis ENDIVLP, MS e RIVAL. Os coeficientes nãopadronizados, Tabela 5, indicam ausência de discriminação para as variáveis EXPRISCO2 e FIRMA, ou seja, estas não influenciam o sinal do retorno sobre o ativo das empresas, confirmando os resultados das duas análises realizadas anteriormente. Na Tabela 9 é apresentada, em ordem de grandeza, a correlação simples entre os preditores e a função. Observa-se que existem pequenas divergências comparadas aos coeficientes padronizados analisados anteriormente. Os centróides dos grupos indicam que o grupo 0 (prejuízo) apresenta valor negativo $(-0,599)$, enquanto o grupo 1 (lucro) apresenta valor positivo $(0,422)$. 


\section{Tabela 9: Matriz Estrutural}

\begin{tabular}{l|c}
\hline \hline \multicolumn{1}{c|}{ Variáveis } & Função 1 \\
\hline EXPRISCO1 (Exposição ao Risco 1) & 0,754 \\
ENDIVLP (Endividamento de Longo Prazo) & 0,332 \\
SETOR (Crescimento do Setor) & 0,241 \\
MS (Participação de Mercado) & 0,238 \\
EXPRISCO2 (Exposição ao Risco 2) & 0,200 \\
RIVAL (Intensidade da Concorrência) & 0,176 \\
POSTURA (Gestão de Custo) & 0,122 \\
FIRMA (Resultado da Empresa) & $-0,068$ \\
SSETOR (Crescimento do Subsetor) & 0,068 \\
DINAM (Dinamismo) & 0,061 \\
\hline \hline
\end{tabular}

Fonte: Dados da pesquisa.

Em relação aos resultados de classificação (Tabela 10), nota-se que o grupo 0 (prejuízo) apresenta maior porcentagem (79\%), se comparado com o grupo 1 (lucro), com 67\%. A percentagem de casos grupados, classificados corretamente, é considerada boa (71,9\%); comparando-a com a percentagem obtida para a análise discriminante dos dados originais $(72,6 \%)$, observa-se pequena diferença entre elas. De acordo com os resultados obtidos, a equação do modelo de análise discriminante para os dados analisados é:

$$
\begin{aligned}
& \text { ROA }=-1,686+0,005 * \text { SSETOR }+0,037 * \text { SETOR }-0,057 * \text { DINAM }+ \\
& 6,751 * \text { RIVAL }++0,998 * \text { POSTURA }+0,467 * \text { EXPRISCO } 1+1,079 * \text { ENDIVLP } \\
& +0,080 * \text { MS }
\end{aligned}
$$

\begin{tabular}{|c|c|c|c|c|c|}
\hline & & & $\mathrm{Gru}$ & ditos & Total \\
\hline & & ROA & 0,00 & 1,00 & 10tal \\
\hline & $\mathrm{N}^{\circ} \mathrm{de}$ & 0,00 & 101 & 27 & 128 \\
\hline Orioinal & IN de casos & 1,00 & 60 & 122 & 182 \\
\hline & $\%$ & 0,00 & 79 & 21 & 100 \\
\hline & & 1,00 & 33 & 67 & 100 \\
\hline
\end{tabular}

Tabela 10: Resultados da Classificação ${ }^{\mathrm{a}}$

a percentagem de casos grupados classificados corretamente: $71,9 \%$

Fonte: Dados da pesquisa.

\section{Análise Discriminante para a Segunda Metade da Amostra}

Com a análise discriminante dos dados da segunda amostra retirada da população, obteve-se, em relação ao Teste de Igualdade de Médias, como variáveis significantes, apenas EXPRISCO1 e ENDIVLP, diferentemente do resultado obtido para os dados originais. 
Na Tabela 11 é apresentada, em ordem de grandeza, a correlação simples entre os preditores e a função. Os centróides dos grupos indicam que o grupo 0 (prejuízo) apresenta valor negativo $(-0,608)$, enquanto o grupo 1 (lucro) apresenta valor positivo $(0,436)$.

\section{Tabela 11: Matriz Estrutural}

\begin{tabular}{l|c}
\hline \hline \multicolumn{1}{c|}{ Variáveis } & Função 1 \\
\hline EXPRISCO1 (Exposição ao Risco 1) & 0,886 \\
ENDIVLP (Endividamento de Longo Prazo) & 0,286 \\
MS (Participação de Mercado) & 0,181 \\
FIRMA (Resultado da Empresa) & $-0,119$ \\
SETOR (Crescimento do Setor) & 0,113 \\
DINAM (Dinamismo) & 0,086 \\
SSETOR (Crescimento do Subsetor) & 0,084 \\
EXPRISCO2 (Exposição ao Risco 2) & $-0,046$ \\
RIVAL (Intensidade da Concorrência) & $-0,036$ \\
POSTURA (Gestão de Custo) & 0,018 \\
\hline \hline
\end{tabular}

Fonte: Dados da pesquisa.

Em relação aos resultados de classificação (Tabela 12), nota-se que o grupo 0 (prejuízo) apresenta maior porcentagem (83\%), se comparado ao grupo 1 (lucro) com $71 \%$. A percentagem de casos grupados, classificados corretamente, é considerada boa $(75,9 \%)$. Comparando-a com a percentagem obtida para a análise discriminante dos dados originais $(72,6 \%)$, observa-se que houve incremento.

\section{Tabela 12: Resultados da Classificação ${ }^{a}$}

\begin{tabular}{|c|c|c|c|c|c|}
\hline & & \multirow[b]{2}{*}{ ROA } & \multicolumn{2}{|c|}{ Grupos Preditos } & \multirow{2}{*}{ Total } \\
\hline & & & 0,00 & 1,00 & \\
\hline \multirow{3}{*}{ Original } & $\mathrm{N}^{\circ}$ de casos & 0,00 & 108 & 22 & 130 \\
\hline & \multirow{2}{*}{0} & 0,00 & 83 & 17 & 100 \\
\hline & & 1,00 & 29 & 71 & 100 \\
\hline
\end{tabular}

a percentagem de casos grupados classificados corretamente: $75,9 \%$

De acordo com os resultados obtidos, a equação do modelo de análise discriminante para os dados analisados é:

$$
\begin{aligned}
& \text { ROA }=-1,119+0,018 * \text { SETOR + 0,002*DINAM + 1,497*RIVAL - } \\
& 0,180 * \text { POSTURA + +0,449*EXPRISCO }+1,299 * \text { ENDIVLP + 0,042*MS }
\end{aligned}
$$

De acordo com os coeficientes não-padronizados da função discriminante, Tabela 3, pode-se verificar que, para todas as análises, as variáveis EXPRISCO2 e 
FIRMA não discriminam a função, ou seja, não existe tendência de influência no sinal da variável dependente ROA (Retorno sobre o ativo).

Comparando os coeficientes padronizados da função discriminante do conjunto de análises realizadas (Tabela 2), identifica-se que, para os dados originais, a variável EXPRISCO1 é o prognosticador mais importante, seguido das variáveis ENDIVLP e MS. Em relação aos dados transformados, a variável mais importante é ENDIVLP, seguida por DINAM e SETOR. Para os dados da $1^{\mathrm{a}}$. e da $2^{\mathrm{a}}$. amostra de análise, tem-se como mais importante a variável EXPRISCO 1, seguida pelas variáveis ENDIVLP e MS, de forma semelhante aos resultados apurados para os dados originais.

De acordo com a Figura 1, o gráfico mostra os dois grupos da variável dependente (ROA) de acordo com seus centróides. Para a elaboração do gráfico foi criada uma nova variável dependente (ROA2), codificada em intervalo de -2 a 2, para se ter melhor visualização gráfica dos dados e uma caracterização da sua distribuição amostral.

Figura 1: Gráfico de Dispersão: Retorno sobre o Ativo versus Função Preditora

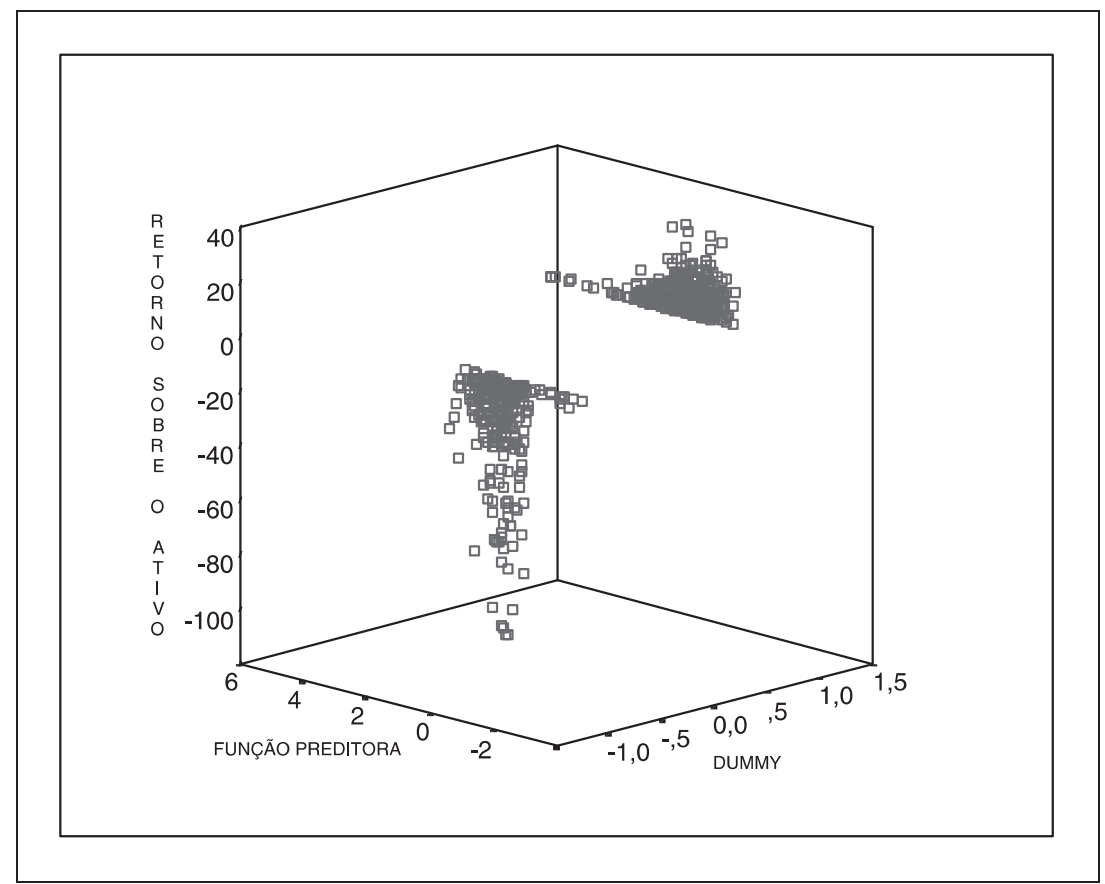

Fonte: elaborada pelos autores. 
Para melhor ajuste da função, foram retirados os outliers do banco de dados. Assim, pode-se notar que o gráfico apresenta os dois grupos analisados $(0=$ prejuízo e $1=$ lucro $)$ e seus respectivos centróides $(-0,586$ e 0,416$)$.

\section{Considerações Finais}

Os objetivos teóricos de verificar as relações entre os determinantes estratégicos e ambientais e os resultados de empresas atuantes em ambientes turbulentos foram satisfatoriamente atendidos, resultando na resposta da questão de pesquisa.

Por meio da aplicação da técnica multivariada de análise discriminante, foram identificados como fatores discriminantes da apuração de lucro ou prejuízo pelas organizações estudadas, as posturas estratégicas de (a) exposição ao risco, expressa pela relação entre o investimento nas atividades operacionais e os resultados financeiros, (b) perfil de endividamento de longo prazo, que representa a relação entre os financiamentos de longo prazo e o total dos financiamentos e (c) participação de mercado, mensurada por meio da relação entre o faturamento bruto anual da organização e o faturamento bruto anual do subsetor.

Os resultados da análise discriminante apontam maior influência da postura de exposição ao risco na determinação do sinal apurado para os resultados financeiros das empresas estudadas, comparativamente às demais variáveis, indicando que o investimento realizado pelos gestores nas atividades operacionais se apresenta como fator determinante de seu resultado.

O peso de baixa intensidade do perfil de endividamento de longo prazo, por apresentar correlação negativa e significativa com a postura de curto prazo, indica a importância do equilíbrio das ações de longo e curto prazo para o resultado organizacional, não apontando a predominância de tal política em relação aos demais fatores estratégicos.

Em relação à participação de mercado, o peso apurado indica que a conquista e a consolidação do espaço no mercado em que atuam permitem às organizações maior previsibilidade de seus resultados, além de ampliar as possibilidades de maior determinação dos rumos e de constituição de áreas de pressão sobre as concorrentes.

Ao analisar conjuntamente os três fatores apresentados anteriormente, podese concluir que os gestores de organizações atuantes em ambiente economicamente turbulento, que adotam uma postura de risco, alocando recursos 
no incremento da capacidade produtiva das empresas, ao invés de acumular lucros, devem buscar a conquista e manutenção de participação de mercado, sempre levando em consideração a necessidade de manter equilíbrio em relação ao perfil de endividamento. Conforme pode ser observado, há predominância dos aspectos relacionados à abordagem da Visão Baseada em Recursos na discriminação entre lucro ou prejuízo dos desempenhos das organizações estudadas, resultado em consonância com os apurados nos trabalhos de Dias (2004), Dias, Gonçalves e Coleta (2004) e Dias e Gonçalves (2005), que apuraram maior influência dos fatores relacionados à gestão de recursos na variação dos resultados das organizações.

Por se tratar da aplicação de um modelo a um setor específico da economia brasileira, propõe-se, como objeto de novos estudos, a aplicação deste a outros setores de atividade, para a ampliação do entendimento dos fatores determinantes da dinâmica dos mercados e sua relação com as políticas estratégicas adotadas pelos gestores, além da proposição de outras variáveis que possam refletir, com maior fidedignidade, as escolhas estratégicas dos gestores, a dinâmica concorrencial das indústrias analisadas e as variações das condições do contexto macroeconômico ocorridas nos períodos analisados.

\section{Artigo recebido em 14.03.2006. Aprovado em 14.02.2007.}

\section{NotA}

${ }^{1}$ Hatten, K. J., \& Schendel, D. E. (1977). Heterogeneity within an industry: firm conduct in the U. S. brewing industry, 1952-1971. Journal of Industrial Economics, 26(2), 97-113.

\section{ReferênCIAS Bibliográficas}

Aaker, D. A.,

Kumar, V., \&

Day, G. S. (2001).

Pesquisa de marketing. São Paulo: Atlas.

Ansoff, H. I. (1975).

Managing strategic surprise by response to weak signals. California Management Review, 18(2), 21-33.
Bandeira-de-Mello, R., \&

Marcon, R. (2004a, setembro).

A mensuração multivariada da 'performance' e suas componentes de variância: uma análise dos efeitos do ano, indústria e firma no contexto brasileiro. Anais do Encontro Nacional da Associação Nacional de Pós-Graduação e Pesquisa em Administração, Curitiba, PR, Brasil, 28. 
Bandeira-de-Mello, R., \&

Marcon, R. (2004b, setembro).

Unpacking firm effects: modeling political alliances in variance decomposition of firm performance in turbulent environments. Anais do Encontro Nacional da Associação Nacional de Pós-Graduação e Pesquisa em Administração, Curitiba, PR, Brasil, 28.

\section{Barney, J. B. (1986).}

Types of competition and the theory of strategy: toward an integrative framework. Academy of Management Review, 11(4), 791-800.

Brito, L.A. L., \&

Vasconcelos, F. C. de (2003a, setembro). How much does country matter? Anais do Encontro Nacional da Associação Nacional de PósGraduação e Pesquisa em Administração, Atibaia, SP, Brasil, 27.

Brito, L.A. L., \&

Vasconcelos, F. C. de (2003b, setembro). Firm performance in an extremely turbulent environment: year, industry and firm effects. Anais do Encontro Nacional da Associação Nacional de Pós-Graduação e Pesquisa em Administração, Atibaia, SP, Brasil, 27.

Brito, L. A. L., \&

Vasconcelos, F. C. de (2004, setembro). Firm performance in Latin America: a different game? Anais do Encontro Nacional da Associação Nacional de Pós-Graduação e Pesquisa em Administração, Curitiba, PR, Brasil, 28.

Child, J. (1974).

What determines organization performance? The universals vs. the it-all-depends. Organizational Dynamics, 3(1), 2-18.

Child, J. (1975).

Managerial and organizational factors associated with company performance - Part II. A contingency analysis. The Journal of Management Studies, 12(1), 12-27.

Chrisman, J. J.,

Hofer, C. W., \&

Boulton, W. R. (1988).

Toward a system for classifying business strategies. Academy of Management Review, 13(3), 413-428.

Dias, A. T. (2004).

Competição, orientação estratégica e desempenho de empresas em ambiente turbulento: uma abordagem empírica. Dissertação de mestrado. Universidade Federal de Minas Gerais, Belo Horizonte, MG, Brasil.

Dias, A. T., \&

Gonçalves, C. A. (2005, junho).

Ambiente competitivo, relações com 'stakeholders' e fatores estratégicos na determinação do desempenho organizacional. Anais do Encontro de Estudos em Estratégia - 3ES, Rio de Janeiro, RJ, Brasil, 2.

Dias, A. T.,

Gonçalves, C. A., \&

Coleta, K. A. P. G. (2004, setembro).

Determinantes estratégicos do desempenho de empresas em ambientes turbulentos. Anais do Encontro Nacional da Associação Nacional de Pós-Graduação e Pesquisa em Administração, Curitiba, PR, Brasil, 28. 
Duncan, R. B. (1972).

Characteristics of organizational environments and perceived environmental uncertainty. Administrative Science Quarterly, 17(3), 313-327.

Hair, J. F.,

Anderson, R. E.,

Tatham, R. L., \&

Black, W. (1998).

Multivariate data analysis (5th ed.). New York: Prentice Hall.

Hawawini, G.,

Subramanian, V., \&

Verdin, P. (2003).

Is performance driven by industry or firm-specific factors? A new look at the evidence. Strategic Management Journal, 24(1), 1-16.

Hunt, S. D. (1997).

Resource-advantage theory: an evolutionary theory of competitive firm behavior? Journal of Economic Issues, 31(1), 59-77.

Hunt, S. D. (2000).

A general theory of competition: resources, competences, productivity, economic growth. Thousand Oaks: Sage Publications Ltd.

Hunt, S. D., \&

Morgan, R. M. (1995).

The comparative advantage theory of competition. Journal of Marketing, 59(2), 1-15.

Hunt, S. D., \&

Morgan, R. M. (1996).

The resource-advantage theory of competition: dynamics, path dependencies, and evolutionary dimensions. Journal of Marketing, 60(4), 107-114.

Hunt, S. D., \&

Morgan, R. M. (1997).

Resource-advantage theory: a snake swallowing its tail or a general theory of competition. Journal of Marketing, 61(4), 74-82.

Kim, L., \&

Lim, Y.(1988).

Environment, generic strategies, and performance in a rapidly developing country: a taxonomic approach. Academy of Management Journal, 31(4), 802-827.

Malhotra, N. K. (2001).

Pesquisa de marketing: uma orientação aplicada (3a ed.). Porto Alegre: Bookman.

Mauri, A. J., \&

Michaels, M. P. (1998).

Firm and industry effects within strategic management: an empirical examination. Strategic Management Journal, 19(3), 211-219.

Miles, R. E.,

Snow, C. C.,

Meyer, A. D., \&

Coleman, H. J., Jr. (1978).

Organizational strategy, structure and process. Academy of Management Review, 3(3), 546-562.

Mingoti, S. A. (2003).

Análise discriminante [Apostila da disciplina estatística multivariada]. Belo Horizonte: ICEX/UFMG. 
Porter, M.E. (1980).

Competitive strategy: techniques for analyzing industries and competitors. New York: Free Press.

Porter, M.E. (1981).

The contributions of industrial organization to strategic management. Academy of Management Review, 6(4), 609-620.
Roquebert, J. A.,

Phillips, R. L., \&

Westfall, P. A. (1996).

Markets vs. management: what 'drives' profitability? Strategic Management Journal, 17(8), 653-664.

Rumelt, R. P. (1991).

How much does industry matter? Strategic Management Journal, 12(3), 167-185.

Schmalensee, R. (1985).

Do markets differ much? American Economic Review, 75(3), 341-351. 